\title{
Some Results on a Double Compound Poisson-Geometric Risk Model with Interference
}

\author{
Dezhi Yan \\ Department of Economic, Shandong Jiaotong University, Jinan, China \\ Email: dezyan@163.com
}

Received November 15, 2011; revised December 10, 2011; accepted December 18, 2011

\begin{abstract}
In this paper, we study the actual operating of an insurance company with random income. A double compound Poisson-Geometric risk model with interference was established. By using the martingale method, the adjustment coefficient equation, the formula and the upper bound of ruin probability, the time to reach a given level in this new risk model were obtained.
\end{abstract}

Keywords: Ruin Probability; Compound Poisson-Geometric Risk Model; Martingale; Stopping Time; Moment Generating Function; Laplace Transform; Adjustment Coefficient Equation

\section{Introduction}

As one of the most important topics in risk theory, the ruin problem in stochastic environments has been studied by many researchers [1,2]. In classical risk model, the claim number process was assumed to be a Poisson process and the individual claim amounts were described as independent and identically distributed random variables. In recent years, the classical risk process has been extended to more practical and real situations. For most of the investigations treated in risk theory, it is very significant to deal with the risks that rise from monetary inflation in the insurance and finance market, and also to consider the operation uncertainties in administration of financial capital.

In order to get more realistic models, the perturbed risk process was introduced by Dufresne and Gerber [3] and investigated by Veraverbeke [4]. The classical risk process perturbed by diffusion is given as follows:

$$
U(t)=u+c t-S_{0}(t)+W(t)
$$

where $u$ is the initial surplus of an insurance company, $c$ $>0$ is the gross premium rate and

$$
S_{0}(t)=\sum_{i=1}^{N(t)} Z_{i}
$$

is the aggregate claim process. $N(t)$ denotes a Poisson process with intensity $\lambda>0 ; Z_{i}, i \geq 1$ is a sequence of independent and identically distributed (i.i.d. for short) nonnegative random variables, independent of $\{N(t) ; t \geq 0\}$. $\{W(t) ; t \geq 0\}$ is standard Brownian motion with $W(0)=0$. Based on the foregoing model, Dufresne and Gerber obtained an integro-differential eq- uation for ruin probability and proved a Lundberg-type inequality corresponding to the ruin probability by means of martingale methods [3]. Gerber and Shiu [5] and Gerber and Landry [6] continued studying the expected discounted penalty function and the time value of ruin. For more details and new developments on the perturbed risk process, the interested readers can refer to [7-13].

Other kinds of generalizations for the classical risk process are inspired by the extensive investigations of both risk and portfolio fluctuations. For instance, the continuous-time risk processes with stochastic interest have been studied by many authors, see [14-21]. Temnov [22] described the premium income by Poisson process and derived an explicit formula for the ruin probability to the corresponding risk process.

Motivated by the above findings, this study aims at gaining an insight into the effects of stochastic premium incomes under perturbation. In this paper, we will consider a double compound Poisson-Geometric risk model with diffussion in which the arrival of policies and claims follows compound Poisson-Geometric process, respectively. Then we study the adjustment coefficient equation, ruin probability and the time to reach a given level.

\section{The Risk Model}

Definition 1 A distribution is said to be Poisson-Geometric distributed, denoted by $P G(\lambda, \rho)$, if it's generating function is

$$
\exp \left\{\frac{\lambda(t-1)}{1-\rho t}\right\}
$$


where $\lambda>0,0 \leq \rho<1$.

Definition 2 Let $\lambda>0$ and $0 \leq \rho<1$, then $\{N(t) ; t \geq 0\}$ is said to be a Poisson-Geometric process with parameters $\lambda, \rho$, if it satisfies

1) $N(0)=0$

2) $\{N(t) ; t \geq 0\}$ has stationary and independent increments;

3) $\forall t>0, N(t)$ is a Poisson-Geometric distributed with parameters $\lambda, \rho$, and

$$
E[N(t)]=\frac{\lambda t}{1-\rho}, \operatorname{Var}[N(t)]=\frac{\lambda t(1+\rho)}{(1-\rho)^{2}} .
$$

Let $U(t)$ denote the surplus at time $t$. Then the double compound Poisson-Geometric risk model with interfereence is defined as

$$
U(t)=u+\sum_{k=1}^{N_{1}(t)} X_{k}-\sum_{k=1}^{N_{2}(t)} Y_{k}+\sigma W(t)
$$

where, $u=U(0)$ is the initial capital, $N_{1}(t)$ is the number of premium up to time $t$, and follows a PoissonGeometric distribution with parameters $\lambda_{1}, \rho_{1} ; N_{2}(t)$ is the number of claims up to time $t$, and follows a Poisson-Geometric distribution with parameters $\lambda_{2}, \rho_{2}$. Let the size or amount of the $k$ th claim be $Y_{k}$ and $\left\{Y_{k}, k \geq 1\right\}$ be a sequence of i.i.d. nonnegative random variables with mean $\mu_{Y}$, variance $\sigma_{Y}^{2}$ and moment generating function $M_{Y}(t) .\left\{X_{k}, k=1,2, \cdots\right\}$ are positive i.i.d. random variables representing the successive premium amounts with mean $\mu_{X}$, variance $\sigma_{X}^{2}$ and moment generating function $M_{X}(t) .\{W(t) ; t \geq 0\}$ is standard Brownian motion with $W(0)=0$ and $\sigma$ is a constant, representing the diffusion volatility parameters. Throughout this paper, we assume that $\left\{N_{1}(t) ; t \geq 0\right\}$, $\left\{N_{2}(t) ; t \geq 0\right\},\left\{X_{k}, k=1,2, \cdots\right\},\left\{Y_{k}, k=1,2, \cdots\right\}$ and $\{W(t) ; t \geq 0\}$ are mutually independent.

In order to ensure the insurance company's stable operation, we assume

$$
E\left[\sum_{k=1}^{N_{1}(t)} X_{k}-\sum_{k=1}^{N_{2}(t)} Y_{k}+\sigma W(t)\right]>0
$$

which implies

$$
\frac{\lambda_{1} \mu_{X}}{1-\rho_{1}}-\frac{\lambda_{2} \mu_{Y}}{1-\rho_{2}}>0
$$

Let

$$
\frac{\lambda_{1} \mu_{X}}{1-\rho_{1}}=(1+\theta) \frac{\lambda_{2} \mu_{Y}}{1-\rho_{2}}
$$

then $\theta>0$ is the relative security loading factor.

For the risk model (1), the time to ruin, denoted by $T$, is defined as

$$
T=\inf \{t \geq 0 \mid U(t) \leq 0\}
$$

and define the ruin probability with an initial surplus $u>0$ by $\psi(u)$, namely

$$
\psi(u)=P\{T<\infty \mid U(0)=u\}
$$

\section{The Property of the Profits Process}

Define the profits process by $R(t)$, i.e.

$$
R(t)=\sum_{k=1}^{N_{1}(t)} X_{k}-\sum_{k=1}^{N_{2}(t)} Y_{k}+\sigma W(t)
$$

It is obviously, we have

$$
\begin{aligned}
E[R(t)] & =\left[\frac{\lambda_{1} \mu_{X}}{1-\rho_{1}}-\frac{\lambda_{2} \mu_{Y}}{1-\rho_{2}}\right] t, \\
\operatorname{Var}[R(t)]= & \operatorname{Var}\left[N_{1}(t)\right] E^{2}\left[X_{K}\right] \\
& +E\left[N_{1}(t)\right] \operatorname{Var}\left[X_{K}\right] \\
& +\operatorname{Var}\left[N_{2}(t)\right] E^{2}\left[Y_{K}\right] \\
& +E\left[N_{2}(t)\right] \operatorname{Var}\left[Y_{K}\right] \\
= & \left\{\frac{\lambda_{1}\left(1+\rho_{1}\right)\left(\mu_{X}^{2}+\sigma_{X}^{2}\right)}{\left(1-\rho_{1}\right)^{2}}+\frac{\lambda_{1} \sigma_{X}^{2}}{\left(1-\rho_{1}\right)}\right. \\
& \left.+\frac{\lambda_{2}\left(1+\rho_{2}\right)\left(\mu_{Y}^{2}+\sigma_{Y}^{2}\right)}{\left(1-\rho_{2}\right)^{2}}+\frac{\lambda_{2} \sigma_{Y}^{2}}{\left(1-\rho_{2}\right)}+\sigma^{2}\right\} t
\end{aligned}
$$

Let

$$
\begin{aligned}
\alpha= & \frac{\lambda_{1} \mu_{X}}{1-\rho_{1}}-\frac{\lambda_{2} \mu_{Y}}{1-\rho_{2}} \\
\beta= & \frac{\lambda_{1}\left(1+\rho_{1}\right)\left(\mu_{X}^{2}+\sigma_{X}^{2}\right)}{\left(1-\rho_{1}\right)^{2}}+\frac{\lambda_{1} \sigma_{X}^{2}}{\left(1-\rho_{1}\right)} \\
& +\frac{\lambda_{2}\left(1+\rho_{2}\right)\left(\mu_{Y}^{2}+\sigma_{Y}^{2}\right)}{\left(1-\rho_{2}\right)^{2}}+\frac{\lambda_{2} \sigma_{Y}^{2}}{\left(1-\rho_{2}\right)}+\sigma^{2}
\end{aligned}
$$

then

$$
\begin{gathered}
E[R(t)]=\alpha t \\
\operatorname{Var}[R(t)]=\beta t
\end{gathered}
$$

Lemma 1 The profits process $\{R(t) ; t \geq 0\}$ has the following properties:

1) $R(0)=0$

2) $\{R(t) ; t \geq 0\}$ has stationary and independent increments.

Theorem 1 For the profits process $\{R(t) ; t \geq 0\}$, there has a function $s=s(r)$ such that

$$
E\left[e^{-r R(t)}\right]=e^{t s(r)}
$$

\section{Proof}




$$
\begin{aligned}
E\left[e^{-r R(t)}\right]= & E\left\{\exp \left[-r\left(\sum_{k=1}^{N_{1}(t)} X_{k}\right)\right]\right\} \\
& \times E\left\{\exp \left[r\left(\sum_{k=1}^{N_{2}(t)} Y_{k}\right)\right]\right\} \times E\left[e^{-r W(t)}\right] \\
= & \exp \left\{t \left[\frac{\lambda_{1}\left(M_{X}(-r)-1\right)}{1-\rho_{1} M_{X}(-r)}\right.\right. \\
& \left.\left.+\frac{\lambda_{2}\left(M_{Y}(r)-1\right)}{1-\rho_{2} M_{Y}(r)}+\frac{1}{2} \sigma^{2} r^{2}\right]\right\}
\end{aligned}
$$

Let

$$
\begin{aligned}
s & =s(r) \\
& =\frac{\lambda_{1}\left(M_{X}(-r)-1\right)}{1-\rho_{1} M_{X}(-r)}+\frac{\lambda_{2}\left(M_{Y}(r)-1\right)}{1-\rho_{2} M_{Y}(r)}+\frac{1}{2} \sigma^{2} r^{2}
\end{aligned}
$$

Theorem 2 The equation

$$
s(r)=0
$$

has a unique positive solution $r=R>0$, and the Equation (6) is said to adjustment coefficient equation of the risk model (1).

Proof From (5), we have

$$
s(0)=0
$$

and since

$$
\begin{aligned}
s^{\prime}(r)= & \frac{\lambda_{1}(\rho-1) M_{X}^{\prime}(-r)}{\left[1-\rho M_{X}(-r)\right]^{2}}+\frac{\lambda_{2}(1-\rho) M_{Y}^{\prime}(r)}{\left[1-\rho M_{Y}(r)\right]^{2}}+r \sigma^{2}, \\
s^{\prime \prime}(r)= & \frac{\lambda_{1} M_{X}^{\prime \prime}(-r)\left(1-\rho_{1}\right)\left[1-\rho_{1} M_{X}(-r)\right]}{\left[1-\rho_{1} M_{X}(-r)\right]^{3}} \\
& +\frac{2 \lambda_{1} \rho_{1}\left(1-\rho_{1}\right)\left[M_{X}^{\prime}(-r)\right]^{2}}{\left[1-\rho_{1} M_{X}(-r)\right]^{3}} \\
& +\frac{\lambda_{2} M_{Y}^{\prime \prime}(r)\left(1-\rho_{2}\right)\left[1-\rho_{2} M_{Y}(r)\right]}{\left[1-\rho_{2} M_{Y}(r)\right]^{3}} \\
& +\frac{2 \lambda_{2} \rho_{2}\left(1-\rho_{2}\right)\left[M_{Y}^{\prime}(r)\right]^{2}}{\left[1-\rho_{2} M_{Y}(r)\right]^{3}} \\
+\sigma^{2}>0, \quad \forall & r>0, \\
s^{\prime}(0) & =-\left[\frac{\lambda_{1} \mu_{X}}{1-\rho_{1}}-\frac{\lambda_{2} \mu_{Y}}{1-\rho_{2}}\right]=-\theta \frac{\lambda_{2} \mu_{Y}}{1-\rho_{2}}<0,
\end{aligned}
$$

we see that $s(r)$ is a convex function on $[0,+\infty)$, and since $s(0)=0, s(0)<0$ and $\lim s(r)=+\infty$, then it can be shown that $s(r)$ has has $\stackrel{r \rightarrow+\infty}{a}$ unique positive solution $r=R$ on $(0,+\infty)$.

For the profits process $\{R(t) ; t \geq 0\}$, let

$$
F_{t}^{R}=\sigma\{R(v) ; v \leq t\} .
$$

Theorem 3 If $r$ and $s$ satisfy the Equation (5), then the surplus $\left\{e^{-r R(t)-t s} ; t \geq 0\right\}$ is a martingale.

Proof

$$
\begin{aligned}
& E\left[e^{-r R(t)-t s} \mid F_{v}^{R}\right]=E\left[e^{-r R(t)-t s(r)} \mid F_{v}^{R}\right] \\
= & E\left[e^{-r R(v)-t s(r)-r[R(t)-R(v)]-(t-v) s(r)} \mid F_{v}^{R}\right] \\
= & e^{-r R(v)-t s(r)} \times E\left[e^{-r[R(t)-R(v)]-(t-v) s(r)} \mid F_{v}^{R}\right] \\
= & e^{-r R(v)-t s}
\end{aligned}
$$

Theorem $4 T$ is a stopping time for $F_{t}^{R}$.

Theorem 5 The probability of the risk model (1) is

$$
\psi(u)=\frac{e^{-R u}}{E\left[e^{-R U(T)} \mid T<\infty\right]}
$$

Corollary $\psi(u) \leq e^{-R u}$.

\section{The Time to Reach a Given Level}

Let

$$
\tau=\inf \{t \geq 0 \mid U(t)=\xi\}
$$

Then $\tau$ is the time when the surplus reaches a given level firstly.

Theorem 6 The Laplace transform of $\tau$ is

$$
E\left[e^{-s \tau}\right]=e^{r \xi}
$$

where $r$ satisfies (6).

Proof For the surplus process $\{U(t) ; t \geq 0\}$, using the theorem of martingale and stopping time, we see that $\tau$ is a stopping time of $F_{t}^{R}$. Let $H(t)=e^{-r R(t)-t s}$, by Theorem 3 the surplus $\{H(t), t \geq 0\}$ is a martingale, hence we have

implying that

$$
E[H(\tau)]=E[H(0)]
$$

$$
E\left[e^{-r R(\tau)-\tau s}\right]=1
$$

Since $R(\tau)=\xi$, so we get

Theorem $7 \quad E[\tau]=\frac{\xi}{\alpha}$ and

$$
E\left[e^{-s \tau}\right]=e^{r \xi} .
$$

$$
\operatorname{Var}[\tau]=\frac{\xi \beta^{2}}{\alpha^{3}}
$$

Proof Using Theorem 6, we have

$$
E\left[e^{-s \tau}\right]=e^{r \xi}
$$

Suppose

$$
\varphi(s)=\ln E\left[e^{-s \tau}\right]
$$

then

$$
\varphi(s)=r \xi
$$

and 


$$
\begin{aligned}
& \varphi^{\prime}(s)=\frac{\mathrm{d} \varphi(s)}{\mathrm{d} r} \cdot \frac{\mathrm{d} r}{\mathrm{~d} s}=\xi \cdot \frac{\mathrm{d} r}{\mathrm{~d} s} \\
& =\xi \cdot \frac{1}{\frac{\mathrm{d} s(r)}{\mathrm{d} r}}=\frac{\xi}{s^{\prime}(r)} \\
& =\frac{\xi}{\frac{\lambda_{1}(\rho-1) M_{X}^{\prime}(-r)}{\left[1-\rho M_{X}(-r)\right]^{2}}+\frac{\lambda_{2}(1-\rho) M_{Y}^{\prime}(r)}{\left[1-\rho M_{Y}(r)\right]^{2}}+r \sigma^{2}} \\
& \text { Let } s=r=0 \text {, then we get } \\
& \begin{aligned}
E[\tau]=- & \left.\frac{\mathrm{d} \varphi(s)}{\mathrm{d} s}\right|_{s=0} \\
= & -\frac{\xi}{-\left(\frac{\lambda_{1} \mu_{X}}{1-\rho_{1}}-\frac{\lambda_{2} \mu_{Y}}{1-\rho_{2}}\right)}=\frac{\xi}{\alpha}
\end{aligned} \\
& \varphi^{\prime \prime}(s)=\frac{\mathrm{d} \varphi^{\prime}(s)}{\mathrm{d} s}=\frac{\mathrm{d} \varphi^{\prime}(s)}{\mathrm{d} r} \cdot \frac{\mathrm{d} r}{\mathrm{~d} s}=\frac{\mathrm{d} \varphi^{\prime}(s)}{\mathrm{d} r} \cdot \frac{1}{s^{\prime}(r)}=\frac{\mathrm{d} \varphi^{\prime}(s)}{\mathrm{d} r} \cdot \frac{1}{s^{\prime}(r)}=-\frac{\xi s^{\prime \prime}(r)}{\left[s^{\prime}(r)\right]^{2}} \cdot \frac{1}{s^{\prime}(r)}=-\frac{\xi s^{\prime \prime}(r)}{\left[s^{\prime}(r)\right]^{3}} \\
& =-\xi\left\{\frac{\lambda_{1} M_{X}^{\prime \prime}(-r)\left(1-\rho_{1}\right)\left[1-\rho_{1} M_{X}(-r)\right]}{\left[1-\rho_{1} M_{X}(-r)\right]^{3}}+\frac{2 \lambda_{1} \rho_{1}\left(1-\rho_{1}\right)\left[M_{X}^{\prime}(-r)\right]^{2}}{\left[1-\rho_{1} M_{X}(-r)\right]^{3}}+\frac{\lambda_{2} M_{Y}^{\prime \prime}(r)\left(1-\rho_{2}\right)\left[1-\rho_{2} M_{Y}(r)\right]}{\left[1-\rho_{2} M_{Y}(r)\right]^{3}}\right. \text {. } \\
& \left.+\frac{2 \lambda_{2} \rho_{2}\left(1-\rho_{2}\right)\left[M_{Y}^{\prime}(r)\right]^{2}}{\left[1-\rho_{2} M_{Y}(r)\right]^{3}}+\sigma^{2}\right\} /\left\{\frac{\lambda_{1}(\rho-1) M_{X}^{\prime}(-r)}{\left[1-\rho M_{X}(-r)\right]^{2}}+\frac{\lambda_{2}(1-\rho) M_{Y}^{\prime}(r)}{\left[1-\rho M_{Y}(r)\right]^{2}}+r \sigma^{2}\right\}^{3}
\end{aligned}
$$

Let $s=r=0$, we have

$$
\operatorname{Var}[\tau]=\left.\varphi^{\prime \prime}(s)\right|_{s=r=0}=\frac{\xi \beta^{2}}{\alpha^{3}}
$$

\section{REFERENCES}

[1] J. Grandell, “Aspects of Risk Theory,” Springer, Berlin, 1991. doi:10.1007/978-1-4613-9058-9

[2] H. U. Gerber, "An Introduction to Mathematical Risk Theory,” Monograph Series, Vol. 8, S. S. Heubner Foundation, Philadelphia, 1979.

[3] F. Dufresne and H. U. Gerber, "Risk Theory for the Compound Poisson Process That Is Disturbed by Diffusion," Insurance: Mathematics and Economics, Vol. 10, 1991, pp. 51-59. doi:10.1016/0167-6687(91)90023-Q

[4] N. Veraverbeke, "Asymptotic Estimations for the Probability of Ruin in a Poisson Model with Diffusion," Insurance: Mathematics and Economics, Vol. 13, 1993, pp. 57-62. doi:10.1016/0167-6687(93)90535-W

[5] H. U. Gerber and B. Landry, "On the Discounted Penalty at Ruin in a Jump-Diffusion and the Perturbed Put Option,” Insurance: Mathematics and Economics, Vol. 22, 1998, pp. 263-276. doi:10.1016/S0167-6687(98)00014-6

[6] H. U. Gerber and E. S. W. Shiu, "On the Time Value of Ruin,” North American Actuarial Journal, Vol. 2, No. 1, 1998, pp. 48-78.

[7] G. J. Wang and R. Wu, "Some Distributions for Classic Risk Process That Is Perturbed by Diffusion,” Insurance: Mathematics and Economics, Vol. 26, No. 1, 2000, pp. 15-24. doi:10.1016/S0167-6687(99)00035-9

[8] G. J. Wang, “A Decomposition of the Ruin Probability for the Risk Process Perturbed by Diffusion,” Insurance: Mathematics and Economics, Vol. 28, No. 1, 2001, pp. 49-59. doi:10.1016/S0167-6687(00)00065-2
[9] C. C.-L. Tsai, "On the Discounted Distribution Functions of the Surplus Process,” Insurance: Mathematics and Economics, Vol. 28, No. 3, 2001, pp. 401-419. doi:10.1016/S0167-6687(01)00067-1

[10] C. C.-L. Tsai, “A Generalized Defective Renewal Equation for the Surplus Process Perturbed by Diffusion," Insurance: Mathematics and Economics, Vol. 30, No. 1, 2002, pp. 51-66. doi:10.1016/S0167-6687(01)00096-8

[11] C. C.-L. Tsai, "On the Expectations of the Present Values of the Time of Ruin Perturbed by Diffusion,” Insurance: Mathematics and Economics, Vol. 32, No. 3, 2003, pp. 413-429. doi:10.1016/S0167-6687(03)00130-6

[12] C. S. Zhang and G. J. Wang, “The Joint Density Function of Three Characteristics on Jump-Diffusion Risk Process,” Insurance: Mathematics and Economics, Vol. 32, No. 3, 2003, pp. 445-455. doi:10.1016/S0167-6687(03)00133-1

[13] S. N. Chiu and C. C. Yin, "The Time of Ruin, the Surplus Prior to Ruin and the Deficit at Ruin for the Classical Process Perturbed by Diffusion,” Insurance: Mathematics and Economics, Vol. 33, No. 1, 2003, pp. 59-66. doi:10.1016/S0167-6687(03)00143-4

[14] J. Paulsen, "Risk Theory in a Stochastic Environment," Stochastic Process and Their Applications, Vol. 21, 1993, pp. 327-361.

[15] J. Paulsen, "Ruin Theory with Compounding Assets: A Survey," Insurance: Mathematics and Economics, Vol. 22, No. 1, 1998, pp. 3-16. doi:10.1016/S0167-6687(98)00009-2

[16] J. Paulsen and H. K. Gjessing, "Ruin Theory with Stochastic Return on Investments," Advances in Applied Probability, Vol. 29, 1997, pp. 965-985. doi:10.2307/1427849

[17] V. Kalashnikov and R. Norberg, "Power Tailed Ruin Probabilities in the Presence of Risky Investments," Sto- 
chastic Process and Their Applications, Vol. 98, 2002, pp. 221-228.

[18] V. E. Bening, V. Yu. Korolev and L. X. Liu, "Asymptotic Behavior of Generalized Risk Processes,” Acta Mathematica Sinica, English Series, Vol. 20, No. 2, 2004, pp. 349-356. doi:10.1007/s10114-003-0244-8

[19] J. Cai, "Ruin Probability and Penalty Functions with Stochastic Rates of Interest," Stochastic Process and Their Applications, Vol. 112, No. 1, 2004, pp. 53-78.

[20] K. C. Yuen, G. J. Wang and W. Ng Kai, "Ruin Probabili- ties for a Risk Process with Stochastic Return on Investments," Stochastic Process and Their Applications, Vol. 110, 2004, pp. 259-274

[21] K. C. Yuen, G. J. Wang and R. Wu, "On the Renewal Risk Process with Stochastic Interest,” Stochastic process and Their Applications, Vol. 116, No. 10, 2006, pp. 14961510.

[22] G. Temnov, "Risk Process with Random Income,” Journal of Mathematical Sciences, Vol. 123, No. 1, 2004, pp. 3780-3794. doi:10.1023/B:JOTH.0000036319.21285.22 